\title{
Editorial
}

\section{E-commerce participation in an extended supply chain: The case of the financial intermediary}

E-commerce is now viewed as an integral part of marketing channels and distribution systems. In an e-enabled supply chain members are able to form digital links to share information, buy, sell and distribute products or services and to transfer cash flow. An e-enabled supply chain is, consequently, perceived as having many advantages, such as increased business efficiency, enhanced information flows, improved transaction speed, wider geographical spread, increased temporal reach, cost reduction and differentiation.

Not only does e-commerce have the potential to enhance supply chain performance, it also has the potential to change traditional supply chain structures. It may even pose a threat to certain supply chain members. The internet, for example, offers a direct link with customers, suppliers and distributors enabling companies to bypass others in the value chain, to control the channel and access to customers.

The term 'disintermediation' describes the bypassing process. Disintermediation occurs when the ultimate supplier of a product or service circumvents intermediaries and sells directly to the consumer; or, when a new intermediary emerges that employs a lower-cost distribution method. The advent of the internet posed a threat of widespread disintermediation, raising concerns over the continued role and importance of intermediaries in a variety of contexts.
Indeed, in many sectors, notably travel, the supply chain has been shortened considerably and consumers regularly deal directly with providers.

In the context of financial services, the intermediary (such as an independent financial advisor) occupies a significant position in the supply chain. He provides not merely a distribution function but, more importantly, specialised information provision, professional advice, customisation to consumers' needs and the reduction of uncertainty. Coordinated and collaborative e-commerce development amongst supply chain members may increase effectiveness and strengthen linkages, but fragmented development may result in business inefficiency and weakened links between supply chain members.

Research conducted by the author and Kathryn Waite at the University of Edinburgh ${ }^{1}$ investigates the potential of the internet to make a difference to the distribution of pensions in the UK. A survey of five thousand independent financial intermediaries (IFAs) was conducted between June-July 2004, from which 692 usable questionnaires were returned.

The survey found that while 99 per cent of IFAs have access to the internet, actual use of the internet for a variety of activities remains rather limited. Main activities tend to support existing relationships rather than the generation of 
new business, and relate more to $\mathrm{B} 2 \mathrm{~B}$ rather than $\mathrm{B} 2 \mathrm{C}$ contexts. The majority of IFAs (63 per cent), for example, do not conduct any pension sales online, although 23 per cent report that they are increasing. In terms of use of the internet (and e-mail) at various stages of the buying process, 27 per cent report using it at the advice stage, 47 per cent at the application stage, 35 per cent for executing a transaction and 64 per cent for servicing an ongoing relationship.

With regards to the extent to which the internet and e-mail have replaced traditional paper-based methods of doing business, the biggest impact has been on the IFA-provider interface where 68 per cent report that the internet has replaced traditional methods. The next biggest impact has been on the relationship with existing clients: 53 per cent and 57 per cent respectively report that it has replaced traditional methods of dealing with existing individual and corporate clients, compared with 42 per cent and 47 per cent respectively for new individual and corporate clients.

In terms of the benefits or outcomes experienced from using the internet for pensions, the greatest impacts reported are: access to research materials (84 per cent), improved service to clients (63 per cent) and time-savings (55 per cent). Forty-two per cent also report experiencing an increase in product administration and business processing, shifting down the chain from providers. Areas where IFAs have experienced limited benefit include an increase in sales revenue (experienced by only 17 per cent) and an increase in the number of new clients (15 per cent). On the positive side, it would appear that only a small proportion have experienced the impact of any disintermediation - only 11 per cent have experienced a reduction in their business as a result of clients dealing directly with providers or felt any weakening of their position within the supply chain.
A key challenge facing the industry is how to widen e-participation. Due to the size and degree of fragmentation of the financial intermediary market, product providers have tended to develop e-commerce relationships with a number of preferred intermediaries, developing proprietary technology and networks. This tailoring of developments to typically larger organisations is argued to be a barrier to e-marketplace participation. Other barriers include the lack of a common framework; misconceptions that the internet has to be a substitute for established methods and not a complementary tool to enhance business; the fact that the industry environment does not encourage innovation; and, that there is little incentive to be the first mover. Moreover, the lack of a common trading platform across the whole financial intermediary sector continues to be a key factor inhibiting the widespread participation of intermediaries in e-commerce.

A large proportion of the financial intermediary sector comprises small and medium-sized enterprises (SMEs). Some of the barriers to participation relate to recognised problems common to SME e-commerce adoption, such as connectivity, while others are more specific to the individual company such as a lack of resources. In contrast, the realisation of the benefits of participation generally rests with the ability of individual SMEs to identify opportunities and to plan their online trading effectively within the constraints of their industry environment.

In terms of what this means for providers, the relationship between larger companies in developing technological relationships is seen as important, not only in capturing a significant part of the marketplace but also in terms of providing an example by which other firms, influenced by a 'me too' development strategy, can follow. Given the 
disadvantages associated with developments tailored to larger or preferred intermediaries; however, an alternative or complementary approach might be to communicate with IT suppliers. A significant proportion of the IFAs surveyed appears to be influenced by the opportunities that arose or emerged to participate in e-commerce provided by external parties, mainly software developers. Rather than attempting to develop a technological relationship directly with intermediaries, a networked approach might prove more beneficial in this context, whereby IT suppliers act as a further intermediary in the technology supply chain between provider and IFA.
This approach does not necessarily have to eliminate individual relationships between providers and intermediaries but may offer a more inclusive approach to e-participation and speed up the rate at which it is adopted.

\section{Tina Harrison Managing Editor}

\section{Reference}

1 The research is part of the 'Pensions online? Producer, distributor and user attitudes and behaviour' project, which is funded by the Economic and Social Research Centre. Further details of the project can be found by visiting the project website: http://webdb.ucs.ed.ac.uk/ management/research/projects/project_entry3.html. 